\title{
Measurement of parasternal intercostal electromyogram during an infective exacerbation in patients with cystic fibrosis
}

\author{
Charles C. Reilly*, Caroline J. Jolley*, Caroline Elston\#, John Moxham* and \\ Gerrard F. Rafferty*
}

\begin{abstract}
The parasternal intercostal muscle electromyogram (sEMGpara) is a measure of neural respiratory drive and reflects lung disease severity in stable cystic fibrosis (CF). The aim of the study was to measure sEMGpara in acute infective exacerbations of $\mathrm{CF}$ and compare changes in sEMGpara with those in conventional lung function measures.
\end{abstract}

12 patients with CF admitted to hospital with an acute chest infection were studied.

There was a significant reduction in mean \pm SD sEMGpara ( $\triangle$ SEMGpara $-38 \pm 19 \%, p<0.001$ ) between admission and discharge. Spirometery also improved significantly from admission to discharge; $\Delta$ forced expiratory volume in $1 \mathrm{~s} \%$ predicted $39 \pm 30 \%, p<0.001$ and $\Delta$ vital capacity \% pred $22 \pm 18 \%, p<0.001$.

sEMGpara has potential value as a nonvolitional measure of change in respiratory function in CF.

\section{KEYWORDS: Chest infection, cystic fibrosis, electromyography}

(n n patients with cystic fibrosis (CF), chronic endobronchial infection and concomitant inflammation of the small airways leads to progressive pulmonary destruction and eventual respiratory failure $[1,2]$. Although a consensus on the definition of an acute pulmonary exacerbation is lacking, symptoms may include breathlessness, malaise, low grade fever, and increased frequency of cough and sputum production. Forced expiratory volume in $1 \mathrm{~s}$ (FEV1) and vital capacity (VC) are the standard measures used to assess changes in lung function during an acute exacerbation [1-4] and the effects of treatments such as antimicrobial therapies, bronchodilators and physiotherapy. FEV1 can be difficult to measure in acutely ill patients as it is dependent on both patient cooperation and effort [4]. Alternative monitoring tools to assess disease severity and the impact of treatment are therefore sought.

Neural respiratory drive (NRD) measured by diaphragm electromyography (EMGdi) provides a sensitive, real time, breath by breath measure of load on the respiratory system [5]. The invasive nature of this technique, however, limits its application. The parasternal intercostal muscles are obligate inspiratory muscles recruited in concert with the diaphragm as ventilation increases [6]. We have previously demonstrated that recording the parasternal intercostal EMG using surface electrodes (sEMGpara) provides a noninvasive alternative to the EMGdi technique [7]. As with EMGdi, sEMGpara provided a measure of the load on the respiratory muscle pump which directly reflects pulmonary function and disease severity in $\mathrm{CF}$ as well as providing a physiological correlate to exercise induced breathlessness [7]. Measurement of sEMGpara could provide a useful technique to assess changes in respiratory function in patients with CF during an infective exacerbation. The advantage of the sEMGpara technique over conventional lung function parameters is that it is nonvolitional.

We hypothesised that sEMGpara can track changes of NRD during an exacerbation of CF. We further hypothesised that the measurement of sEMGpara may be used to monitor respiratory disease status in unstable CF patients. The aim of the study was, therefore, to investigate the feasibility and potential usefulness of measuring sEMGpara in patients with CF during an acute infective episode and compare the measurements to standard lung function.

\section{METHODS}

\section{Subjects}

Patients with CF, admitted to King's College Hospital (London, UK) with an acute exacerbation of their lung disease were studied. The study was approved by the King's College Hospital research ethics committee and all participants provided informed, written consent.
AFFILIATIONS

*King's College London, and

\#King's College Hospital NHS Foundation Trust, London, UK.

CORRESPONDENCE

C.C. Reilly

King's College London

Division of Asthma, Allergy and Lung

Biology

Dept of Asthma, Allergy and

Respiratory Science

King's College London School of Medicine

Bessemer Road

London

SE5 9PJ

UK

E-mail: charles.c.reilly@kcl.ac.uk

Received:

Sept 192011

Accepted after revision:

Dec 082011

First published online:

Jan 202012

European Respiratory Journal Print ISSN 0903-1936 Online ISSN 1399-3003 
The clinical diagnosis of an acute exacerbation was determined by a CF specialist physician; patients presented with increased cough, volume and purulence of sputum, tachypnoea, dyspnoea, reduced exercise tolerance and a decrease in pulmonary function (FEV1 \% predicted) compared with their best FEV1\% pred in the last 12 months [8].

\section{Pulmonary function}

FEV1 and slow VC [9] were measured in all patients in the seated, upright position at the patient's bedside using a portable electronic spirometer (Vitalograph Gold Standard ${ }^{\circledR}$; Vitalograph $_{\circledast}$, Buckingham, UK).

\section{Electromyographic measurements}

sEMGpara was recorded using bipolar surface electrodes (Kendall Arbo ${ }^{\circledR}$, Tyсо healthcare ${ }^{\circledR}$, Neustadt, Germany) placed bilaterally $3 \mathrm{~cm}$ from the midpoint of the sternum in the second intercostal space. The positive electrode was placed on the right side of the chest and the reference electrode on the lateral aspect of the right clavicle $[10,11]$. Prior to placement of the surface electrodes the skin was thoroughly cleaned with abrasive gel (Nuprep $^{\mathrm{TM}}$ abrasive skin gel; Weaver and Company, Aurora, CO, USA). The area was swabbed with an alcohol wipe to ensure that no residual gel was left on the skin (Sterets ${ }^{\mathrm{TM}}$ preinjection swabs, Medlock, UK) [12, 13]. Measurements of sEMGpara were performed at the bedside with the patient in an upright, seated position with the arms supported.

The electromyogram (EMG) signals were amplified and bandpass filtered between $10 \mathrm{~Hz}$ and $3 \mathrm{kHz}$ (RA- $8_{\mathbb{B}}$ biomedical amplifier; Yinghui Medical Tech Ltd® , Guangzhou, China) and acquired and displayed on a laptop computer (MacBook; Apple Computer Corp, Cupertino, CA, USA) running Chart ${ }_{\circledR}$ version 5.4 software (ADInstruments ${ }^{\circledR}$, Colorado Springs, CO, USA) with analogue to digital sampling at $2 \mathrm{kHz}$ (Powerlab ADInstruments $\mathbb{R}$ ). Post-acquisition bandpass filtering between $20 \mathrm{~Hz}$ and $1 \mathrm{kHz}$ was applied to all recordings using the acquisition software. Peak root mean square (RMS) per breath was calculated and averaged over $1 \mathrm{~min}$.

To allow for comparison between patients and with other studies the resting peak RMS sEMGpara per breath averaged over 1 min measured on discharge was normalised to the EMG signal obtained during a maximal volitional manoeuvre also performed at discharge. Four different maximal manoeuvres were performed: inspiratory capacity, maximal static inspiratory effort against an occluded airway, maximal sniff and maximal voluntary ventilation (MVV) for $15 \mathrm{~s}$. The MVV was only performed once. The other respiratory manoeuvres were repeated at least five times and, irrespective of manoeuvre, the numerically largest EMG was used for normalisation. We have previously demonstrated that this is a reproducible method of normalising sEMGpara signals [7].

\section{Study protocol}

sEMGpara and spirometery measurements were performed within $48 \mathrm{~h}$ of admission and on the day of discharge. The maximal volitional manoeuvres to normalise the sEMGpara recording were performed on the day of discharge only. Any adverse effects or comments from the patients regarding the measurement technique were also noted.
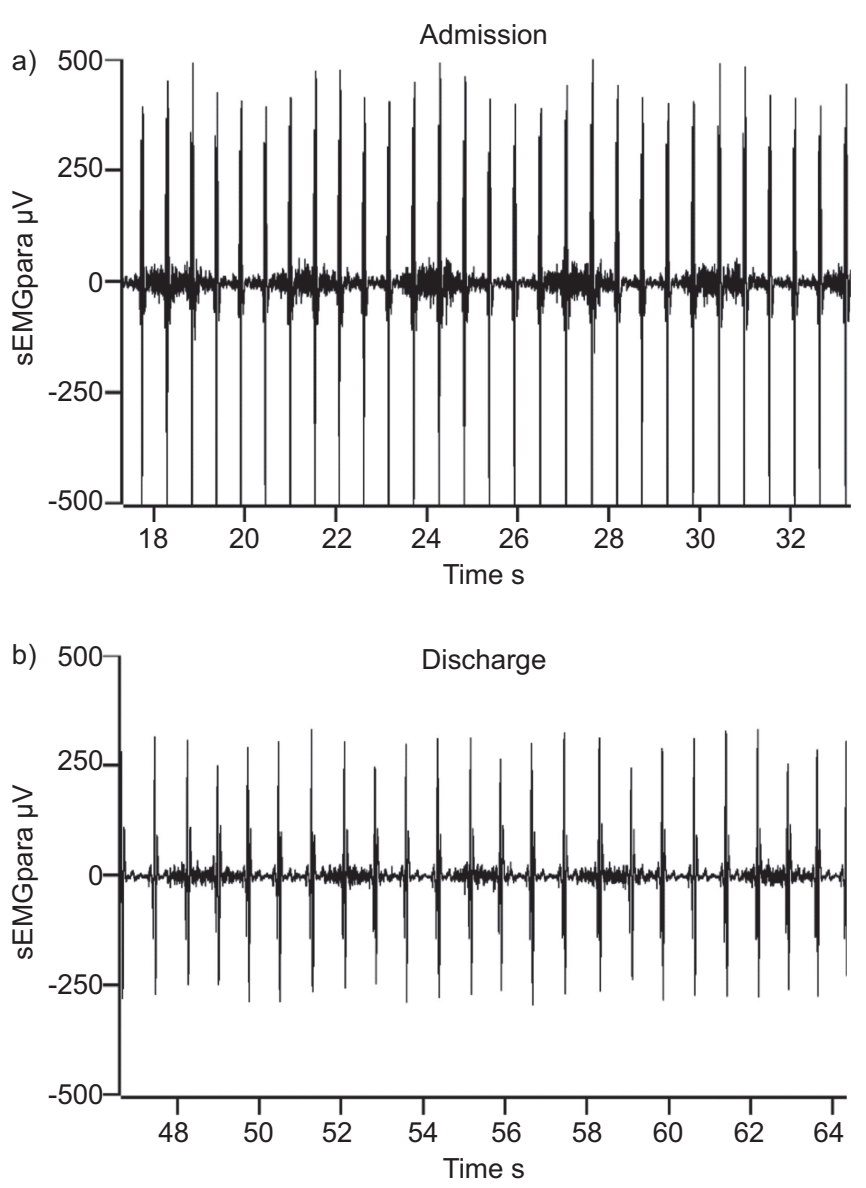

FIGURE 1. Surface parasternal electromyogram (sEMGpara) recorded in a patient with cystic fibrosis (forced expiratory volume in 1 s $20 \%$ pred) on a) admission and b) discharge from hospital following treatment for an infective exacerbation of their lung disease.

\section{Data analysis}

All data with the exception of arterial oxygen saturation $\left(\mathrm{Sa}, \mathrm{O}_{2}\right)$ and length of stay were normally distributed and therefore expressed as mean $\pm \mathrm{SD}$. $\mathrm{Sa}_{\mathrm{O}_{2}}$ is reported as median (range). Differences in variables between admission and discharge were assessed using paired t-tests for the normally distributed data. Differences in $\mathrm{Sa}_{1} \mathrm{O}_{2}$ were assessed using the Mann-Whitney test. The association between $\triangle \mathrm{sEMG}$ ara and $\triangle \mathrm{FEV} 1 \%$ pred was examined using Pearson correlation coefficients.

\section{RESULTS}

12 patients with CF (mean \pm SD age $23 \pm 4$ yrs, 4 female) took part in the study. The median (range) length of stay was 8 (522) days. Clear, phasic, inspiratory activity of the parasternal intercostal muscle was obtained in all subjects at both time points (fig. 1). There was a significant reduction in mean $\pm \mathrm{SD}$ sEMGpara ( $\Delta$ sEMGpara $38 \pm 19 \%, \mathrm{p}<0.001)$ measurements between admission and discharge. No adverse measurement effects were noted. Patients reported that they liked the measurement technique as it was simple to perform.

The reductions in sEMGpara were associated with improvements in FEV1 $(\Delta \mathrm{FEV} 1 \%$ pred $39 \pm 30 \%, \mathrm{p}=0.001), \mathrm{VC}(\Delta \mathrm{VC} \%$ pred $22 \pm 18 \%, \mathrm{p}=0.004)$ and peak expiratory flow $(\mathrm{PEF})(\Delta \mathrm{PEF}$ 


\begin{tabular}{|c|c|c|c|c|c|c|c|c|c|c|c|c|}
\hline TABLE 1 & $\begin{array}{l}\text { Dem } \\
\text { chan } \\
\text { for a }\end{array}$ & $\begin{array}{l}\text { ogra } \\
\text { ges i } \\
\text { n act }\end{array}$ & $\begin{array}{l}\text { hic, lung } \\
\text { individua } \\
\text { te exacerb }\end{array}$ & $\begin{array}{l}\text { unction a } \\
\text { patient r } \\
\text { ation }\end{array}$ & $\begin{array}{l}\text { sEMGp } \\
\text { asured }\end{array}$ & $\begin{array}{l}\text { a measur } \\
\text { iables be }\end{array}$ & $\begin{array}{l}\text { d in cys } \\
\text { ween adr }\end{array}$ & $\begin{array}{l}\text { fibrosis } \\
\text { sion an }\end{array}$ & $\begin{array}{l}\text { atients } \\
\text { dischars }\end{array}$ & $\begin{array}{l}\text { the day } \\
\text { from he }\end{array}$ & $\begin{array}{l}f \text { admissior } \\
\text { bital followi }\end{array}$ & $\begin{array}{l}\text { and the } \\
\text { treatment }\end{array}$ \\
\hline 1 & 20 & $\mathrm{~F}$ & 22 & 15.5 & 62 & 37 & 77 & 20 & 375 & 12 & 51 & -83 \\
\hline 3 & 21 & M & 16 & 20 & 39 & 21 & 69 & 0 & 350 & 25 & 19 & -25 \\
\hline 4 & 20 & $M$ & 8 & 22 & 20 & 120 & 38 & 71 & 370 & 5 & 18 & -43 \\
\hline 5 & 20 & M & 5 & 20 & 43 & 5 & 62 & 10 & 400 & 0 & 18 & -30 \\
\hline 6 & 23 & $\mathrm{~F}$ & 12 & 15 & 56 & 41 & 74 & 22 & 320 & 25 & 29 & -38 \\
\hline 7 & 21 & M & 6 & 17 & 32 & 28 & 72 & 10 & 270 & 37 & 18 & -35 \\
\hline 8 & 25 & $M$ & 14 & 16 & 24 & 33 & 42 & 24 & 260 & 27 & 14 & -9 \\
\hline Mean $\pm S D$ & $22 \pm 4$ & & $8(5-22)^{\#}$ & $20 \pm 3$ & $41 \pm 17$ & $39 \pm 30$ & $64 \pm 17$ & $22 \pm 18$ & $338 \pm 70$ & $22 \pm 12$ & $19 \pm 11$ & $-38 \pm 19$ \\
\hline
\end{tabular}

sEMGpara: surface parasternal electromyogram; BMI: body mass index; FEV1: forced expiratory volume in $1 \mathrm{~s}$; \% pred: \% predicted; VC: vital capacity; PEF: peak expiratory flow; $A$ : admission; $F$ : female; $M$ : male. ${ }^{*}$ : length of stay is expressed as median (range).

$22 \pm 12 \%, p=0.003)$ between admission and discharge in all patients (table 1 and fig. 2). The \% increase in FEV1\% pred was not statistically different from the reductions observed in sEMGpara $\mu \mathrm{V}$.

There was considerable individual variability in the change in FEV1\% pred (range 5-120\%) and the corresponding change in sEMGpara measurements (range -9- -83\%) (table 1). Reflecting this variability, there was no correlation between the increase in FEV1 \% pred and the fall in sEMGpara between admission and discharge $(r=0.13, p=0.69)$.

There was no significant difference in median (range) $\mathrm{Sa}_{1} \mathrm{O}_{2}$ between admission and discharge (95 (92-99) versus 95 (93-99), $\mathrm{p}=0.30$ ). The mean $\pm \mathrm{SD}$ resting level of $\mathrm{sEMGpara} \%$ max was $11 \pm 4 \%$ on the day of discharge.

\section{DISCUSSION}

The main finding of the current study was that during successful hospital treatment for acute infective exacerbations of $\mathrm{CF}$ there was a reduction in NRD, as measured by parasternal intercostal muscle EMG activity with a corresponding improvement in lung function (FEV1 and VC). For the group of 12 patients the overall improvement in sEMGpara $(\mu \mathrm{V})$ was similar to the improvement in FEV1 \% pred; there was, however, no correlation between these two variables.

\section{Critique of the methods}

Surface EMG recordings often have poor between subject and between occasion reproducibility, due to variations in electrical contact and electrode position relative to the underlying muscle. We have previously demonstrated reproducible between occasion parasternal EMG signals measured during resting tidal breathing, with a mean inter-occasion coefficient of variation of $0.05 \pm 0.04 \mu \mathrm{V}$ [7]. Using Bland and Altman analysis MAARSINGH et al. [11] reported good reproducibility of sEMGpara measured during resting tidal breathing in young adults and both healthy and asthmatic school children. They recorded a mean difference for all the groups of $1 \mu \mathrm{V}$ or less.

Under resting conditions tidal breathing is principally under autonomic control, and hence sEMGpara provides a nonvolitional measure of NRD. Raw non-normalised sEMGpara therefore provides a measure of improvement in clinical status in individual patients and can be used to monitor changes in respiratory load over time.

Normalising the sEMGpara signal to that obtained from a maximal manoeuvre (sEMGpara \% max), although requiring patient cooperation, provides a relative measure of disease severity and allows comparison between CF patients and other studies [7]. In the current study, sEMGpara measurements at discharge were normalised using maximal manoeuvres performed at discharge; procedures to normalise sEMGpara on admission were not performed as we felt the ability of acutely unwell patients to produce maximal volitional efforts was likely to be impaired. Not normalising sEMGpara does not diminish the use of this technique as a monitoring tool to assess change in respiratory disease status.

The use of surface electromyography to record respiratory muscle activity has been criticised because of difficulties in separating postural activity of the trunk muscles from that of the breathing muscles [14]. To minimise EMG contamination from the muscles of the trunk, all measurements were performed in the relaxed, upright seated position, with the patients' arms and trunk supported. Overt postural muscle EMG contamination during the parasternal EMG recordings was not observed $[15,16]$. EMG signals can also be contaminated by background electrical noise [17]. In the current study this was overcome by using shielded cables and a $50 \mathrm{~Hz}$ notch filter on the EMG amplifier. The amplifier was also connected to one of the electrical earthing points on the ward. 

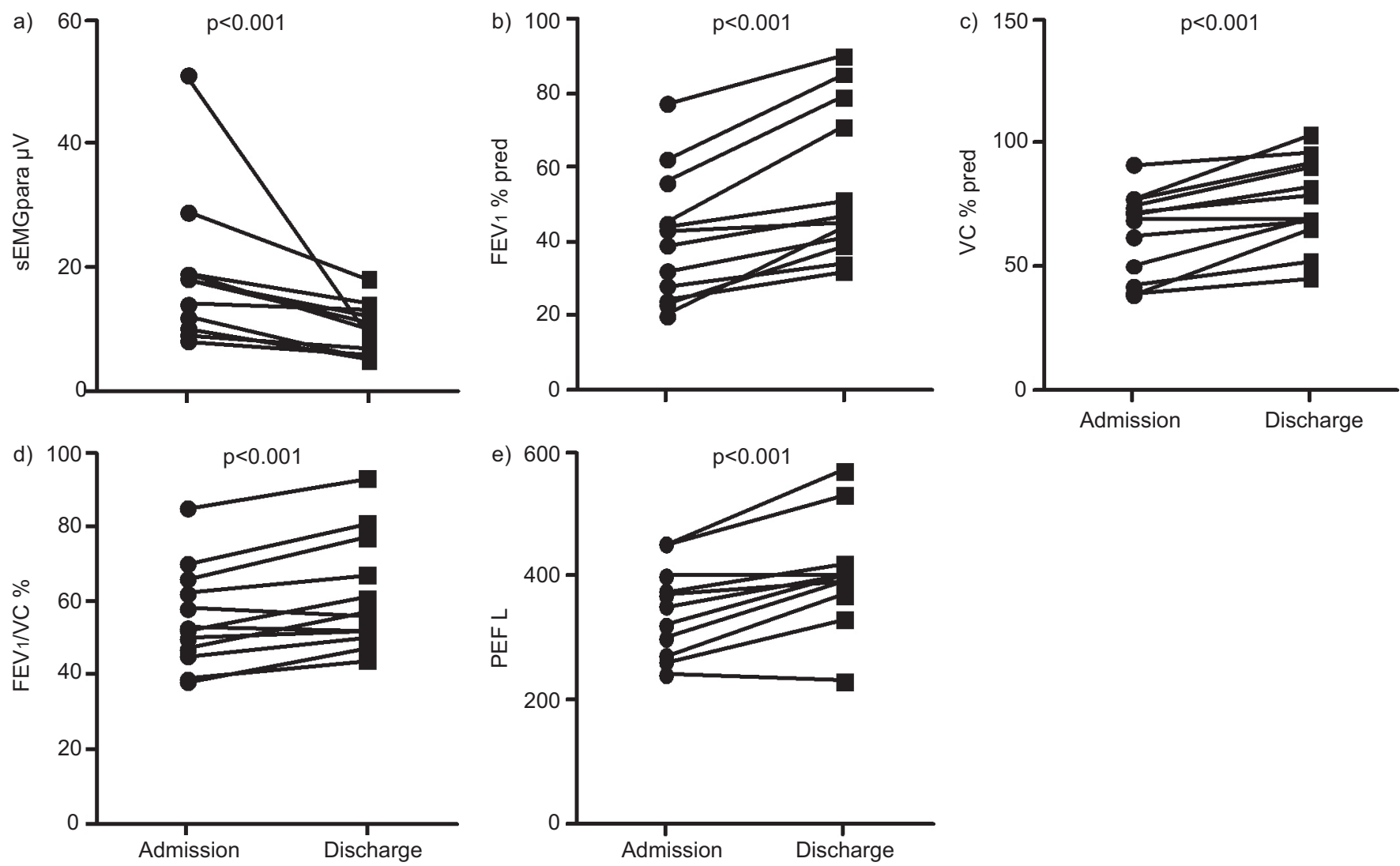

FIGURE 2. Individual changes in a) surface parasternal electromyogram (sEMGpara) $\mu \mathrm{V}$, b) forced expiratory volume in $1 \mathrm{~s}(\mathrm{FEV} 1) \%$ pred, c) vital capacity (VC) \% pred, d) FEV $1 / N C$, and e) peak expiratory flow (PEF). Each line represents an individual patient.

Electromyography using surface electrodes is also affected by the thickness of subcutaneous fat. None of the CF patients studied were overweight and no change in body mass index was observed during their admission.

\section{Significance of the findings}

There was a significant decrease in sEMGpara $(\mu \mathrm{V})$ following the treatment and resolution of the infective episode in the patients. The fall in sEMGpara reflected a fall in the respiratory drive required to maintain ventilation at levels appropriate for adequate gas exchange, and was most likely a result of combined reductions in airway obstruction, hyperinflation, and a change in chest wall configuration.

Even though there was a 39\% mean reduction in sEMGpara following the resolution of the infective episode, the resting level of sEMGpara \% max on discharge was much higher than that previously reported in young healthy individuals $(5.8 \pm 3 \%)$ [7]. This increased level of NRD as measured by sEMGpara \% max was in keeping with that previously reported in CF [7], asthma [18] and chronic obstructive pulmonary disease [19].

sEMGpara could be of potential value in monitoring $\mathrm{CF}$ lung disease. As sEMGpara measures the overall load on the respiratory muscle pump it may better reflect lung disease severity than individual pulmonary function tests, which only measure one aspect of lung pathology, e.g. FEV1 as a measure of airway obstruction. Similarly other respiratory variables such as respiratory rate do not provide directly quantifiable measures of load on the respiratory system and work of breathing and can be influenced by emotional state.

sEMGpara provides a novel complementary method to assess improvements in respiratory status in patients with $\mathrm{CF}$ following an acute exacerbation of their chest disease. The method is nonvolitional and provides a quantifiable breath by breath measure of the load on the respiratory muscle pump. Moreover, sEMGpara could provide a monitoring technique in circumstances where conventional spirometry is difficult to perform or inappropriate, such as in patients with severe acute respiratory failure, severe haemoptysis, severe breathlessness, and intractable cough [20].

In some of the patients the change in sEMGpara was greater than the change in FEV1 \% pred, reflecting the poor relationship between FEV1 and other pathophysiological changes in pulmonary mechanics (hyperinflation, intrinsic positive endexpiratory pressure, dynamic compliance, ventilation/perfusion mismatch, hypoxia and hypercapnia) which occur in $\mathrm{CF}$ lung disease. In other patients the change in sEMGpara was smaller than the change in FEV1 \% pred. The clinical status of the patients at admission may have resulted in submaximal FEV1 efforts and account for the larger observed improvements in FEV1. Future studies could further explore this hypothesis by comparing daily changes in FEV1 and sEMGpara to better understand the sensitivity of these measures or by applying an alternative, nonvolitional test of pulmonary function, such as impulse oscillometry or multiple breath inert gas washout. The 
logistics of applying such tests in acutely unwell patients are at present however limited.

\section{Conclusion}

sEMGpara provides a noninvasive measure of NRD in patients with CF. Potentially sEMGpara may be a useful nonvolitional monitoring tool in patients with $\mathrm{CF}$ to assess changes in ventilatory mechanics and load following an infective exacerbation of their chest disease. sEMGpara provides a complementary measure to conventional lung function in acutely unwell $\mathrm{CF}$ patients. Normalisation of the sEMGpara recordings (sEMGpara $\%$ max) allows for comparison between different patients and patient groups. Subsequent studies should explore the sensitivity of sEMGpara measurements compared with conventional lung function tests, both in the acute setting and long-term monitoring, and for assessing responses to therapeutic interventions.

\section{SUPPORT STATEMENT}

C.C. Reilly is supported by a Medical Research Council (London, UK) PhD studentship.

\section{STATEMENT OF INTEREST}

None declared.

\section{REFERENCES}

1 Gibson RL, Burns JL, Ramsey BW. Pathophysiology and management of pulmonary infections in cystic fibrosis. Am J Respir Crit Care Med 2003; 168: 918-951.

2 Goss $\mathrm{CH}$, Burns JL. Exacerbations in cystic fibrosis: epidemiology and pathogenesis. Thorax 2007; 62: 360-367.

3 Burns JL, Ramsey BW, Smith AL. Clinical manifestations and treatment of pulmonary infections in cystic fibrosis. Adv Pediatr Infect Dis 1993; 8: 53-66.

4 Ramsey BW, Boat TF. Outcome measures for clinical trials in cystic fibrosis. Summary of a Cystic Fibrosis Foundation consensus conference. J Pediatr 1994; 124: 177-192.

5 Jolley CJ, Luo Y-M, Steier J, et al. Neural respiratory drive in healthy subjects and in COPD. Eur Respir J 2009; 33: 289-297.

6 De Troyer A, Legrand A, Gevenois PA, et al. Mechanical advantage of the human parasternal intercostal and triangularis sterni muscles. J Physiol 1998; 513: 915-925.
7 Reilly CC, Ward K, Jolley CJ, et al. Neural respiratory drive, pulmonary mechanics and breathlessness in patients with cystic fibrosis. Thorax 2011; 66: 240-246.

8 Gold R, Carpenter S, Heurter H, et al. Randomized trial of ceftazidime versus placebo in the management of acute respiratory exacerbations in patients with cystic fibrosis. J Pediatr 1987; 111: 907-913.

9 Miller MR, Hankinson J, Brusasco V, et al. Standardisation of spirometry. Eur Respir J 2005; 26: 319-338.

10 Duiverman ML, van Eykern LA, Vennik PW, et al. Reproducibility and responsiveness of a noninvasive EMG technique of the respiratory muscles in COPD patients and in healthy subjects. J App Physiol 2004; 96: 1723-1729.

11 Maarsingh EJ, van Eykern LA, Sprikkelman AB, et al. Respiratory muscle activity measured with a noninvasive EMG technique: technical aspects and reproducibility. J App Physiol 2000; 88: 1955-1961.

12 Wong Y-M, Ng GYF. Surface electrode placement affects the EMG recordings of the quadriceps muscles. Phys Ther Spor 2006; 7: 122-127.

13 Rainoldi A, Melchiorri G, Caruso I. A method for positioning electrodes during surface EMG recordings in lower limb muscles. J Neurosci Methods 2004; 134: 37-43.

14 Sinderby C, Friberg S, Comtois N, et al. Chest wall muscle cross talk in canine costal diaphragm electromyogram. J Appl Physiol 1996; 81: 2312-2327.

15 Gorini M, Spinelli A, Gigliotti F, et al. Changes in neural drive (EMGd) and neuromuscular coupling during histamine-induced bronchoconstriction in patients with asthma. Eur Respir J 1988; 1: 691-697.

16 Sprikkelman AB, Van Eykern LA, Lourens MS, et al. Respiratory muscle activity in the assessment of bronchial responsiveness in asthmatic children. J Appl Physiol 1998; 84: 897-901.

17 Reaz M, Hussain M, Mohd-Yasin F. Techniques of EMG signal analysis: detection, processing, classification and applications. Biol Proced Online 2006; 8: 11-35.

18 Steier J, Jolley CJ, Polkey MI, et al. Nocturnal asthma monitoring by chest wall electromyography. Thorax 2011; 66: 609-614.

19 Murphy PB, Kumar A, Reilly C, et al. Neural respiratory drive as a physiological biomarker to monitor change during acute exacerbations of COPD. Thorax 2011; 66: 602-608.

20 ATS. Standardization of Spirometry, 1994 Update. American Thoracic Society. Am J Respir Crit Care Med 1995; 152: 1107-1136. 